\title{
SOBRE LO MÍSTICO EN LO ESTÉTICO Y SOBRE LO ESTÉTICO EN LO Místico EN LA ObRa de ERNST Tugendhat*
}

\author{
Ángela Uribe Botero \\ Universidad Nacional de Colombia \\ auribeb@unal.edu.co
}

\section{R E S U M E N}

Los textos de Ernst Tugendhat sobre mística están acompañados de una serie de referencias a la experiencia estética. Dadas estas referencias y la importancia que puede atribuirse a ellas en el propósito de Tugendhat de aclarar el sentido de lo místico, en este trabajo exploro la posibilidad de responder positivamente a esta pregunta: ¿Puede la experiencia estética comportar algunos de los rasgos de la experiencia mística? El punto de partida en mi propósito será atender a la reflexión de Tugendhat en torno al término "asombro" en el contexto de la experiencia estética. A continuación doy las razones por las cuales, según el autor, la expresión "iqué asombroso que exista el mundo!" tiene, en efecto, el sentido que Wittgenstein negó para ella. Concluyo mostrando que quien, con este tipo de expresiones, afirma la belleza del mundo no solo expresa la emoción que comporta el asombro; expresa también una disposición para asumir una actitud mística.

PALABRAS CLAVE

Ersnt Tugendhat, experiencia mística, egocentricidad, experiencia estética, asombro.

\section{A B S T R A C T}

Ernst Tugendhat's works on mysticism are provided of a series of references to the aesthetic experience. Based on these references, and the significance they may be attributed in Tugendhat's purpose to clarify the sense of mysticism, in this paper I explore the possibility to answer affirmatively the question: Can the aesthetic experience imply some of the traits attributed to the mystical experience? My starting point to get the answer is to focus on Tugendhat's reflections on the term "astonishment" in the context of the mystical experience. I then give the reasons that, according to Tugendhat and opposed to Wittgenstein, may make us think that the expression ' How astonishing that the world exists!' has indeed a sense. Finally, I conclude showing that anyone using expressions like this to affirm the beauty of the world, not only expresses the emotion implied by his astonishment: he also expresses a disposition to assume a mystical attitude.

KE Y W O R D S

Ersnt Tugendhat, mystical experience, egocentricity, aesthetic experience, astonishment.

* Agradezco a Ignacio Ávila y a Raúl Meléndez por sus comentarios a una versión de este texto. 


\section{SOBRE LO MÍSTICO EN LO ESTÉTICO Y SOBRE LO ESTÉTICO} EN LO Místico EN LA OBRA DE ERNST TUGENDHAT

\section{$\mathrm{M}$}

i propósito en este texto es ampliar el sentido dado por Ernst Tugendhat (2004) a las siguientes palabras: "Cualquier frase como: "iqué asombroso que exista $A$ »! es transparente en dirección a lo místico" (p. 178) ${ }^{1}$. El contexto en el que aparecen estas palabras en Egocentricidad y mística, a mi manera de ver, no es lo suficientemente amplio como para responder a las preguntas que ellas suscitan. Dicho contexto es el de la delimitación del concepto de asombro $\mathrm{y}$, más precisamente, el de la objeción contra Wittgenstein, que constituye el punto de partida de dicha delimitación. Para Tugendhat, y contra lo que Wittgenstein afirma, es al parecer posible dar sentido a las formas como expresamos nuestro asombro ante la existencia del mundo. Según Wittgenstein, el asombro por la existencia del mundo y, junto con él, la dimensión emocional que comporta, no pueden ser expresados significativamente. Esto se explica porque, para él, uno no podría imaginarse que el mundo no exista y, entonces, asombrarse de que, en efecto, el mundo existe es ininteligible. Una frase como "iqué asombroso que exista el mundo!" no tiene pues sentido (Wittgenstein, 1999). Pero ¿son estas afirmaciones de Wittgenstein realmente indiscutibles? (Tugendhat, 2004, p. 172). El hecho de que no lo sean se relaciona, para Tugendhat, justamente con la posibilidad de hacer explícita, a través de una expresión, la dimensión emocional que comporta el asombro ante la existencia del mundo.

El punto de partida en mi propósito en este texto será reconstruir la reflexión de Tugendhat en torno al sentido de las palabras "iqué asombroso que exista A!" en el contexto del asombro ante la presencia de un objeto bello ${ }^{2}$. A continuación hago una

\footnotetext{
${ }^{1}$ En el original: Jeder solche Satz wie «wie erstaunlich daß es A gibt» is dursichtig auf das Mystishe (Tugendhat, 2003, p. 160).

${ }^{2}$ Según Tugendhat, podemos experimentar otras formas de asombro compara-
} 
descripción de la forma como, según él, la frase "iqué asombroso que exista el mundo!" tiene, en efecto, sentido. Lo anterior dará paso a las siguientes preguntas: ¿Qué debemos entender por el término "transparente" en la frase "«iqué asombroso que exista A!» es transparente en dirección a lo místico"? Dado una determinada forma de entender la palabra "transparente" en este contexto, ¿podría acaso pensarse que la experiencia mística puede ser descrita como una experiencia estética? Y, más aun, ¿podría acaso pensarse que la experiencia estética comporta rasgos de la experiencia mística?

Mi propósito de ampliar el sentido de las palabras citadas solamente se cumple si se da una respuesta afirmativa a estas preguntas. Doy, entonces, las razones por las cuales creo que una descripción de la experiencia mística podría muy bien venir acompañada de afirmaciones que hagan alusión a la belleza del mundo. Para concluir, doy las razones por las que creo, incluso, que la experiencia estética comporta una disposición para la actitud mística.

\section{Asombro}

En su texto "Asombro" Tugendhat (2004, pp. 167-179) busca aclarar el sentido de la expresión "iqué asombroso que exista el mundo!" por la vía de aclarar el sentido de la expresión "iqué asombroso que exista A!". En frases como esta última se expresa, según Tugendhat (2004), una emoción, vinculada, a su vez, al hecho de que algo es digno de nuestra atención, aun cuando ese algo no nos resulta útil (p. 173). Esta forma de explicar el sentido de las palabras "iqué asombroso que exista A!" es apenas una definición formal del término "asombro", y es a la que el autor

bles a esta. Sin embargo, dado que en este texto me interesa ser explícita en relación con la forma como podría establecerse para él un vínculo entre lo estético y lo místico, me limito a lo que el autor dice acerca del asombro ante la belleza de un objeto. (Tugendhat, 2004, pp. 176-177). 
prefiere atenerse para desarrollar su posición en relación con la de Wittgenstein.

Cuando nos asombramos por algo, cuando nos asombramos por el hecho de que algo exista, vemos a ese algo como destacado, como notable, en medio de las demás cosas. Aquello que despierta en nosotros la emoción del asombro es, entonces, digno de nuestra atención. Estamos ante una obra de arte y nos vemos impelidos a afirmar "iqué asombroso que exista este cuadro!", "iqué asombroso que exista el Autorretrato de Rembrandt!", por ejemplo ${ }^{3}$. Entre otros cuadros que hay en el museo, en la sala en la que se exponen algunas obras de Rembrandt, uno de sus autorretratos nos parece especialmente notorio. Nos acercamos a él como atendiendo a una suerte de llamado. Ante la presencia de ese cuadro, dice Tugendhat (2004), "uno es interpelado de dos maneras" (p. 175). En primer lugar, la mirada de Rembrandt en el autorretrato parece emplazarnos. Los ojos son grandes, oscuros y muy delicadamente pintados sobre el lienzo. Los trazos para darles forma aparecen muy finos y hay brillo en esos ojos. Las pupilas se ven dilatadas, y hay, entonces, vida en ellos. Si la tenemos de frente, la mirada de Rembrandt, tal como aparece en el cuadro, parece fijarse en la nuestra, como si quisiera decirnos algo. La delicadeza en los trazos de colores casi transparentes que dan forma a los ojos contrasta con las pinceladas de oleo bruscas y gruesas que configuran el resto de la cara en el Autorretrato. Uno es interpelado por el cuadro de una segunda manera que responde, según Tugendhat (2004), a su calidad estética (p. 175). Quizás el autor se refiera con esto último al uso muy perfeccionado de los colores, a la composición del cuadro y a la maestría en el dibujo. Quizás esto remita, a su vez, a la pregunta acerca de cómo fue posible que alguien elaborara un objeto tan perfecto; es decir, a la

\footnotetext{
${ }^{3}$ En las siguientes líneas me tomo la libertad de ampliar la descripción de las razones por las que, creo, Tugendhat (2004) utiliza el ejemplo del Autorretrato de Rembrandt para hacer claridad sobre el sentido de la expresión "iqué asombroso que exista A!" (p. 175).
} 
pregunta acerca de cómo llega a hacer parte del mundo un objeto con esas características.

En su texto sobre el asombro Tugendhat (2004) parece sugerir que el hecho de ser interpelados de una forma $u$ otra por una obra de arte hace que nos sumerjamos en ella (p. 175). Nos sumergimos en algo, en primer lugar, porque la existencia de ese algo nos resulta notable. Como si no pudiéramos pasar de largo por el hecho de que ese algo existe; en términos de Tugendhat (2004), no podemos pasar de largo por el hecho de que ese algo ocupa un lugar en el mundo (p. 178). Una vez allí, es decir, una vez que hemos respondido a esa suerte de llamado que nos hace lo notable, permanecemos, si se quiere, con él, a su lado, para atender a la forma como él se destaca.

Quizás sea a esto a lo que se refería Kant (1983) cuando en su Crítica del juicio, y hablando sobre la representación de un objeto como un objeto bello, decía: "[lo bello es] aquello con lo cual la imaginación puede jugar sin haberlo buscado... nos es siempre nuevo y no nos [cansamos de mirarlo]"4 (§ 22, B 72|A71).

En esta frase, a mi parecer, se destacan dos aspectos a los que hace referencia Tugendhat en su definición formal del asombro: en primer lugar, se destaca el carácter prolongado de la dimensión emocional que se impone con el objeto y que lo hace distinto de la simple sorpresa ${ }^{5}$ : un objeto bello nos es siempre nuevo y no nos cansamos de mirarlo. No nos cansamos de mirarlo porque tan pronto atendemos a él, alguno de sus aspectos, o varios de ellos, llaman nuestra atención, una y otra vez, y lo hacen de modo que cada uno de ellos pareciera ser visto como por primera vez: la

\footnotetext{
${ }^{4}$ Las palabras entre corchetes remiten a la traducción de Manuel García Morente del original: "... das, womit die Einbildungskraft ugesucht... spielen kann, und jederzeit neu, und man wird seines Einblickes nicht überdrussig” (§22, B72|A71). (Kant, 1977, p.144).

${ }^{5}$ En las páginas 174 y 175 de su ensayo Tugendhat deja ver la diferencia entre la sorpresa y el asombro. Esta diferencia tiene que ver con el tiempo prolongado durante el cual es experimentado el asombro.
} 
mirada profunda de Rembrandt, una vez, los trazos negros que dan una forma casi perfecta a su camisa, otra vez; los golpes de pincel aparentemente desordenados y que dan forma a su cara, una vez más. El segundo de los aspectos que se destaca en la frase de Kant y que, me parece, está también presente en la definición formal que Tugendhat da del asombro es el hecho de que este es una emoción independiente de la posibilidad de instrumentalizar el objeto al que atendemos; es decir, es independiente de la posibilidad de convertirlo en algo que cumpla para nosotros un propósito. Esta última es una de las razones por las cuales en la Crítica del juicio Kant dice que ante la presencia de un objeto al que llamamos "bello" la imaginación juega libremente: entre otras cosas, no está atada a la constricción que le impone el hecho de que el objeto preste algún servicio a quien lo observa.

La presencia de una obra de arte, dice Tugendhat (2004, p.175), puede resultar cautivadora, incluso estremecedora. Sentirse cautivado por un objeto parece ser parte de la emoción que configura al asombro, y parece responder al hecho de que dado que permitimos que ese objeto genere afecto en nosotros, entonces "no nos cansamos de mirarlo". Uno de los efectos de esa especie de cautiverio en el que nos pone a vivir, por ejemplo, una obra de arte que llama nuestra atención es que ya no estamos, en rigor, ante un objeto sino, justamente, con él: nuestra manera de atender a él, que, como dice Tugendhat, nos cautiva y nos estremece, crea, como lo veo, una especie de vínculo de intimidad entre quien observa y el objeto. Una vez más, de nuestra manera de atender al objeto bello es constitutivo el hecho de que experimentamos un estado emocional que se vive en la forma de sentirse uno replegado en ese objeto (i.e."no nos causamos de mirarlo").

Bien puede, sin embargo, ocurrir que no nos cansemos de atender cuidadosamente al Autorretrato porque estamos exclusivamente interesados en saber cómo llegó a existir ese objeto. Queremos saber, por ejemplo, quién era Rembrandt, qué tipo de técnica empleó para mezclar los colores que dieron lugar a la palidez del rostro representado; queremos reconocer en sus trazos 
la influencia de otro de los pintores de la época; queremos, en fin hacer del objeto un objeto para nuestro conocimiento. En efecto, en esta disposición para saber cosas sobre el objeto atendemos con cuidado a él, nos detenemos durante un tiempo prolongado en los detalles que lo constituyen y, sin embargo, puede ser que el asombro, esto es, la impronta emotiva en nuestro atender a él, no esté presente. De allí resulta, para Tugendhat (2004), que "[hay] un factor emocional que diferencia a la sorpresa y al asombro del mero prestar atención" (p. 176).

Verse cautivado por la presencia de un objeto y atender a él una y otra vez, sin que esa forma de atender se agote en las preguntas por las razones que tienden a explicar su existencia o en las preguntas por la utilidad que puede prestarnos, es, entonces, experimentar la emoción del asombro. Sin embargo, desde el punto de vista de Tugendhat (2004), el asombro es una emoción que no necesariamente deja de sentirse tan pronto como se hayan encontrado las razones que explican el hecho de que el objeto ocupa un lugar en el mundo. Es más, Tugendhat advierte que esas explicaciones pueden hacer que la emoción del asombro perdure en el tiempo o aumente (p. 176). Lo distintivo en el asombro parece ser, pues, que el objeto que nos causa asombro llama nuestra atención, no en primer lugar porque no sepamos nada sobre él y queramos ampliar nuestro conocimiento, sino porque su existencia nos parece inconcebible; porque su existencia suscita en nosotros una emoción. Es la existencia misma del objeto, y no nuestra ignorancia acerca de las razones de esa existencia, la que despierta nuestro asombro, dice Tugendhat (2004). De allí que la proposición "iqué asombroso que exista A!" sea para él una proposición existencial que "no nos lleva a preguntar por una razón" (p. 176). A lo que estamos enfrentados, pues, ante la belleza de un objeto no es a nuestra falta de comprensión sobre él; no es a nuestra falta de comprensión sobre las razones por las que él ocupa un lugar en el mundo.

Para aclarar más precisamente el sentido del asombro ante la presencia de un objeto bello, quizás valga la pena preguntarse por 
la forma como nos vemos a nosotros mismos, dado el hecho de que estamos con ese objeto. Tugendhat ha dicho: un objeto bello nos interpela, nos cautiva, hace que nos sintamos estremecidos. Estas tres cosas quizás hagan que nuestra relación con el objeto hable, si se quiere, acerca de nosotros, de la forma como nos vemos, dado que nos parece asombroso el hecho de que él exista. Si un objeto nos cautiva de este modo, nos vemos pequeños. Si llama la atención el hecho de que A exista, hasta el punto de exclamar "¡qué asombroso que exista A!", es porque en el momento en el que estamos con A solo A (y no B, C, o D) llama nuestra atención. A se destaca entre los demás. A parece grande en relación con todo lo demás, incluidos nosotros mismos. Tanto como el espectáculo del Autorretrato de Rembrandt, por ejemplo, cause en nosotros la emoción del asombro y, entonces, nos veamos impelidos a afirmar "iqué asombroso que exista este cuadro!", estamos de alguna manera expresando el hecho de que nosotros mismos no nos destacamos o no tenemos la intención de parecer destacados entre las cosas que nos rodean. Dado el Autorretrato, cedemos el paso a una forma de grandeza que nos sobrecoge para, si se quiere, habitarla; cedemos en nuestra acostumbrada propensión a ocupar el centro; en nuestra acostumbrada propensión a atribuirle importancia a aquello entre lo cual normal y cotidianamente nos desenvolvemos: nuestros deseos, nuestras metas, nuestras obligaciones. Haciendo esto terminamos por vernos en nuestra pequeñez, en lo insignificante que parecemos nosotros mismos y todas las cosas a las que solemos atribuirles importancia.

\section{“¿QUÉ ASOMBroso QUE EXISTA EL MUNDo!”}

El sentido para la frase "iqué asombroso que exista el mundo!" se relaciona, en primer lugar, con el hecho de que con ella (como con "iqué asombroso que exista A!") se expresa también una emoción. Una vez más, la emoción responde a la circunstancia de que consideramos que es inconcebible que exista el mundo. Como con A, ocurre también, en el caso de lo asombroso que 
puede llegar a parecernos el hecho de que el mundo exista, que esta forma de ser del asombro es lo que convierte al mundo en el centro de nuestra atención (Tugendhat, 2004, p. 168). Dicho así, esto suena extraño. ¿Cómo puede constituirse el mundo en centro de nuestra atención? Y ¿en qué consiste, más explícitamente, este "prestar atención al mundo"?

Para responder a estas preguntas es preciso tener en cuenta, con Tugendhat, que el hecho de que el mundo pase a ser el centro de nuestra atención presupone una actitud a la que Tugendhat llama "mística". ¿Cómo llegamos a asumirla? La actitud mística es para Tugendhat una, entre otras ${ }^{6}$, de las respuestas al hecho doloroso de que solemos vernos dispersos entre lo que queremos y la contingencia en relación con las posibilidades de obtener eso que queremos.

Lo que esencialmente caracteriza a la voluntad humana es la conciencia de la radical insuficiencia de la propia capacidad de acción para hacer realidad no sólo ciertas esperanzas, sino metas muy elementales: la vida, la salud, la alimentación, estar con otros. Los seres humanos anticipan como posibles las desgracias con que animales de otras especies tan sólo se enfrentan cuando tienen lugar, de modo que durante toda la vida tienen conciencia de la tensión entre una realización y una decepción de la que no son responsables, y en consecuencia temen siempre frustraciones y desgracias. (Tugendhat, 2004, p. 137).

Quien asume una actitud mística sabe reconocer el lugar de la contingencia en el cumplimiento de sus metas. Voluntariamente se dispone, entonces, a liberarse de la avidez con la que suele estar atado a ellas; quiere, así, evitar el dolor que comporta la tensión entre sus metas particulares y el hecho de que el cumplimiento de ellas no depende de él (Tugendhat, 2004, pp. 132, 134; 2007, pp. 160-164); lo hace en la forma de darles a esas metas y a esos

\footnotetext{
${ }^{6}$ La religión es una de ellas (Tugendhat, 2004, pp. 136 y ss.).
} 
deseos menos importancia. Tanto como crea que esos deseos y esas metas pueden llegar a ser constitutivos de lo que él es, lo hace en la forma de darse a sí mismo menos importancia (Tugendhat, 2004, p. 163).

Bien puede uno darse a sí mismo menos importancia de un modo parcial; es decir, reconociendo que ahí están también los otros dándose importancia, que ahí están ellos procurando el éxito en la consecución de sus propias metas y deseos. En esa medida, uno puede relativizarse a sí mismo cediendo ante el hecho de que quizás y en ocasiones las metas o los deseos de los otros pueden tener prioridad sobre los propios (Tugendhat, 2004, p. 163). Quien así se dispone para ceder en favor de las metas de los otros da un paso atrás y centra su atención, por lo menos parcialmente, en ellas. Esta forma de relativizarse puede ser vista como un paso "fluido", dice Tugendhat (2004), hacia lo místico: "En todo apartarse del centro en favor de otros hay un rasgo místico" (p. 163). Sin embargo, en la relativización de los propios deseos y de las propias metas en favor de las de los demás hay la posibilidad de que el bienestar de los otros sea visto como constitutivo del propio bienestar y, por lo tanto, de las propias metas y de los propios deseos. Con esto último se mantiene aún la condición de la que quiere ser liberado el místico: la tensión dolorosa que comporta el hecho de tener metas y la conciencia de que su cumplimiento no depende de él. De allí que la forma como tiende a restarse importancia a sí mismo el místico sea más exigente en relación con aquello de lo cual quiere desprenderse. Su tranquilidad, lo sabe, dependerá de la medida en que consiga desplazar el centro de su atención de sí mismo, en general.

En términos de la forma como se orienta la atención, lo que tiene lugar tanto en la actitud mística como en la actitud dispuesta para ver un objeto bello, es una suerte de sustitución. El místico y el esteta no solamente apartan la atención de sí mismos; en cuanto lo hacen, ella tiene que ser puesta en otro lugar: A, los otros, lo otro (Tugendhat, 2004, p. 163). En el caso de la actitud mística, lo otro, los otros, puede ser entendido como todo aquello que no 
somos nosotros y a lo que llamamos "el mundo" dada la actitud mística, puede, entonces, constituirse en el centro de nuestra atención (Tugendhat, 2004, p. 132).

¿En qué consiste prestar atención al mundo? Antes de responder a esta pregunta es preciso aclarar la forma como, según Tugendhat, es concebido el mundo desde la perspectiva del místico. En la historia de la humanidad hay diversas manifestaciones de la mística ${ }^{8}$. A cada una de ellas corresponde cierta forma de concebir el mundo, de la que deriva, a su vez, cierta forma de "prestar atención al mundo" (Tugendhat, 2007, pp. 162-167). La menos cargada de teoría y, con ello, la más clara forma de prestar atención al mundo es, para Tugendhat, aquella en la que el uso de la palabra "mundo" no hace énfasis en él como una cosa o como un conjunto de cosas, sino como "todo lo demás"; justamente, como todo aquello que no es uno mismo. El mundo al que presta atención el místico desde esta perspectiva, dice Tugendhat (2007), "es algo puramente formal, como el espacio, no es una instancia independiente" (p. 166). Dicho esto, y como lo veo, la forma como el místico se relaciona con el mundo puede ser puesta en los siguientes términos: se trata de verse uno, no frente al conjunto de todas las cosas, sino con ellas, entre todas ellas. Tan pronto como el místico se ve a sí mismo como uno más entre todo lo demás y con todo lo demás experimenta aquello que Tugendhat (2004), acudiendo a Rudolf Otto, llama "un estado de ánimo específicamente numinoso" (p. 134). Lo numinoso es caracterizado por Otto en términos de lo misterioso, lo atemorizante, lo atrayente. De la descripción que hace Otto de lo numinoso que Tugendhat (2004) destaca dos características: "«lo más poderoso», «lo incomparablemente grande» ante lo cual uno se siente

\footnotetext{
${ }^{7}$ En alguna ocasiones, y para referirse a lo mismo a lo que se refiere empleando el término "mundo", Tugendhat (2004) usa el término "universo" (pp. 132-133).

${ }^{8}$ Este hecho no se relaciona con la actitud mística, derivada de la motivación y que da origen a todas las manifestaciones históricas de la mística; se relaciona, justamente, con la historia y con particularidades culturales correspondientes.
} 
pequeño" (p. 134). El verse uno a sí mismo con todo lo otro y entre todo lo otro comporta, pues, una dimensión emocional: el mundo puede llegar a atemorizar, no tanto por su tamaño, sino por la forma desacostumbrada como desplaza tan radicalmente nuestra atención de nosotros mismos a él. La grandeza del mundo se refiere, como lo entiendo, al hecho de que todo eso que ahora es convertido en espectáculo pasa a ocupar el centro de nuestra atención. En lo que sigue precisaré estas afirmaciones.

El estado de ánimo específicamente numinoso al que se refiere Tugendhat remite a la forma como los seres humanos, una y otra vez, hacemos distinción entre lo grande y lo pequeño. Normalmente cuando hacemos este tipo de contrastes, lo hacemos de un modo en el que la grandeza o la pequeñez de algo son relativas a otra cosa. Uno mismo puede parecer más grande o más pequeño que otras cosas, en muchos sentidos. Sin embargo, hay una única instancia en la que definitivamente no nos vemos como más pequeños en comparación con el tamaño de algo determinado; es más, en ella solo nos vemos pequeños. Nos vemos pequeños, entre otras razones, porque aquello con ocasión de lo cual nos vemos así no puede ser instrumentalizado (Tugendhat, 2004, p. 136); no puede ser puesto a nuestra disposición.

En cuanto alguien asume una actitud mística, el mundo está ahí ante todo como un espectáculo asombroso. Dada la impronta emotiva que comporta el asombro, esta forma de aparecer del mundo hace que sea visto como por primera vez. El hecho de ver el mundo como asombroso es señal de que el místico ha cedido, ha dado un paso atrás. Ha decidido atribuirle importancia al mundo hasta permitir que sea este el que ocupe el centro. Y sin embargo, él mismo —el místico- está también entre aquello que él mismo no es, está con todo eso.

Si el místico se dispone para restarse importancia, para restarle importancia a aquello en lo cual se configuran sus deseos y sus expectativas, no podrá más que relacionarse con el mundo de tal modo que este no responda a esos deseos y a esas expectativas. Esto se aclara mejor aludiendo al sentido en el que el místico, 
dada la actitud para la que se dispone, está con todo eso a lo que llamamos "el mundo"; no está frente a él. Si la relación del místico con el mundo no se define a partir de sus disposiciones volitivas, o incluso de sus disposiciones teóricas - como cuando queremos hacer uso de las cosas del mundo o cuando queremos conocerlo-, su modo de vincularse al mundo no es otro que aquel propio del estar entre todo, con todo lo que ve o imagina. A falta de una relación instrumental o puramente teórica con el mundo, y dado el espectáculo que puede llegar a constituir esta forma de verse con las cosas del mundo - entre ellas-, en la forma de verse ante el hecho simple de que el mundo existe, el místico afirmará "iqué asombroso que exista el mundo!".

Para aclarar mejor el sentido de esta frase, y teniendo en cuenta lo dicho por el autor sobre la frase "iqué asombroso que exista A!", es preciso aún ahondar más en el sentido en el que, según Tugendhat, el místico presta atención al mundo. ¿Se relaciona acaso este "prestar atención al mundo" con la manera como habla Kant acerca de la forma como una obra de arte bella "nos es siempre nueva y no nos cansamos de mirarla"?

Si la existencia del mundo puede llegar a parecernos misteriosa, entonces puede parecernos también asombrosa. Dada la manera como el mundo llega a presentársele al místico, este se ve, si se quiere, emplazado por él. En términos metafóricos, es como si el mundo reclamara la atención del místico. Tan pronto como esto ocurre, el mundo aparece para él como lo nunca antes visto, como lo visto por primera vez. La respuesta del místico a lo asombroso del mundo es la misma respuesta del esteta al Autorretrato: una vez que cierta forma de emplazamiento ha tenido lugar, el místico se detiene en el mundo para prestarle atención.

La forma como el místico presta atención al mundo comporta, de un modo decisivo, una dimensión emocional que, como vimos en el caso del esteta, se relaciona con lo que Tugendhat (2004) llama "el límite de lo que puede ser explicado" (p. 176). Ante el misterio que ha llamado su atención, bien puede querer el místico resolver las preguntas que le suscita dicho misterio: ¿cómo llegó 
a ser el mundo? ¿Acaso fue creado por alguien? ¿Quién es ese alguien? Si el mundo no fue creado por alguien, entonces, ¿en qué momento de la historia pasada llegó a ser y cómo ocurrió este llegar a ser del mundo? No es esto, sin embargo, lo decisivo en la relación que el místico construye con el mundo en la forma de prestar atención a él. No es este el tipo de enfrentamiento con el mundo - llamémoslo teórico- el que tiene lugar, en principio, cuando se ha asumido una actitud mística. De hecho, y como lo he dicho, la actitud mística no dispone a quien la asume a ninguna forma de enfrentamiento. Se trata, una vez más, de un estar con el mundo que suscita una emoción. De esta emoción es constitutivo ese verse pequeño místico, incomparablemente pequeño ante lo incomparablemente grande (Tugendhat, 2004, p. 135), no por su tamaño, sino porque es incomparablemente otro, es decir, incomparablemente "no yo".

Es importante aclarar que la mística no se acaba, según Tugendhat (2004), en llevar a cabo determinadas actividades, como meditar. Tampoco es el resultado de ser, de cierta manera, un iniciado en alguna práctica particular. "Me esfuerzo por ver la mística como algo abierto a todos los seres humanos, como algo que no depende de que se hagan ciertos ejercicios ni de que se posea una constitución especial" (p. 132). Teniendo esto en cuenta, junto con lo dicho unas líneas más arriba, podríamos concluir parcialmente lo siguiente: 1 . Aun cuando puede estar vinculada causalmente con ella, la manera como describe Tugendhat este "prestar atención al mundo" no es teórica ni objetivizante. Es decir, aun cuando el místico quiera saber cómo es el mundo, aun cuando quiera, además, comportarse teóricamente en relación con él, este "querer saber cómo es el mundo" no tiene la dimensión emocional que define al asombro y que acompaña una forma de prestar atención exclusivamente mística. El místico puede ser cualquiera. Esto es, cualquiera que haya asumido la actitud de restarse importancia y, con ella, se permita verse desde el mundo, es decir, de un modo en que aparezca con las cosas del mundo, entre ellas, no frente a ellas. 


\section{SOBRE LO MÍSTICO EN LO ESTÉTICO}

Hasta aquí he presentado solo algunas de las características negativas de la forma como, según Tugendhat, el místico presta atención al mundo, dado que ha asumido una actitud. He dado, sin embargo, algunos pasos que sirven para develar el sentido de la frase "iqué asombroso que exista el mundo!" a la luz de la frase "iqué asombroso que exista A!". De lo dicho podría concluirse que la palabra "transparente" en la frase "«iQué asombroso que exista A!» es transparente en dirección a lo místico" se relaciona con el sentido en el que se entienden el asombro y el prestar atención, tanto en "iqué asombroso que exista A!" como en "iqué asombroso que existe el mundo!"; se relaciona con el hecho de que en las dos frases el sentido para "asombro" y para "prestar atención" es el mismo. ¿Es solo a esto a lo que se refiere Tugendhat cuando usa el término "transparente" en la frase citada? Tanto como así sea podría quizás decirse que, según Tugendhat, hay rasgos de lo estético en lo místico. En este sentido, como lo he sugerido arriba, la forma de ser numinosa del mundo podría llegar a ser comparable con la forma como es asombrosa para nosotros la existencia de una obra de arte. Dada la grandeza de todo lo otro, en la actitud mística podemos llegar a sentirnos pequeños de un modo que no es relativo a nada. Esto último también puede ser comparable con el modo en que nos vemos descentrados - como pequeños - ante la presencia del Autorretrato. Como vimos, el Autorretrato nos cautiva, nos estremece y hace que le restemos importancia a lo que nos ocupa cotidianamente.

Quizás pueda decirse, sin embargo, un poco más del sentido en el que Tugendhat habla de "transparente" en esta frase. La experiencia que vive el místico en relación con mundo no es solo "transparente" en el sentido de "comparable" con la experiencia de quien se ha permitido asistir al espectáculo en el que puede consistir una obra de arte. Dado que el sentido para "asombro" y para "prestar atención", tanto en el caso de la experiencia estética como en el caso de la experiencia mística, es, como vimos, el mis- 
mo, los dos tipos de experiencia no son, creo, solo comparables. Es decir, quizás Tugendhat no se valga del ejemplo del Autorretrato solamente para ilustrar, contra Wittgenstein, el sentido que, en efecto, puede tener la frase "iqué asombroso que exista el mundo!". Quizás el ejemplo sea una forma de mostrar, más bien, que "en todo apartarse del centro en favor de otros hay un rasgo místico" (Tugendhat, 2004, p. 163).

Esta última frase aparece en el contexto en el que Tugendhat caracteriza la actitud mística, es decir, la disposición para restarse uno mismo importancia. Tal frase no aparece en los contextos en los que él, en ocasiones, describe la experiencia mística. Sin embargo, a mi manera de ver, dicha frase puede ser leída teniendo en cuenta este contexto. Esto significaría, como lo veo, que en el apartarse del centro para el que nos dispone la emoción del asombro ante un objeto bello hay un rasgo místico. Significa que en todo sentirse cautivado por un objeto, en todo sentirse estremecido y pequeño, dado que él existe, en todo sentirse de este modo, más allá de la posibilidad de instrumentalizar ese objeto y más allá de la posibilidad de conocerlo, en fin, en todo "ya no ser el centro" hay un rasgo místico. Tanto como pueda decirse esto, ¿podría decirse también que la experiencia mística comporta rasgos estéticos? ¿Habría entonces lugar a la siguiente paráfrasis?: "En todo apartarse del centro en favor de los otros y en favor de lo otro hay un rasgo estético". ¿Por qué no? Claramente, no puede decirse que para Tugendhat la experiencia mística es reductible a la experiencia estética: las ocasiones en las que nuestro asombro se despierta para prestar atención son en los dos casos distintas. De esto, sin embargo, no se sigue que la experiencia mística no comporte un rasgo estético.

De la mano de Tugendhat he tratado de aclarar por qué determinados objetos dan lugar en nosotros a la expresión "iqué asombroso que exista A!". He dado las razones por las que esto ocurre. Esos mismos objetos suelen dar lugar en nosotros a frases como "A es bello" por las mismas razones. Con "A es bello", como vimos, se expresa algo semejante, si no lo mismo - y por las 
mismas razones - que se expresa con "iqué asombroso que exista A!". En esta medida, ante el Autorretrato y asombrados, justamente, porque el objeto existe, afirmamos su belleza; afirmamos, si se quiere, la emoción que provoca el ver la grandeza del objeto, el hecho de que es él, y no nosotros mismos, el que ahora ocupa el centro de nuestra atención. En los términos en los que Kant habla acerca de las características del juicio estético, "A es bello" puede querer decir: "A produce en mi cierta emoción que hace que no me canse de mirar a A", y entonces "iqué asombroso que exista A!".

¿Podría acaso pensarse que lo mismo ocurra con el mundo? Es decir, la emoción que despierta en alguien que se dispone a habitar el mundo de la forma descrita arriba podría expresarse no solamente con las palabras "iqué asombroso que exista el mundo!", sino también con un genuino "iqué bello que es el mundo!". Hay un modo como esta última frase puede llegar a expresar ese restarle importancia a nuestros asuntos particulares del que he hablado para caracterizar lo místico. Me refiero al hecho de que quien afirma la belleza del mundo no expresa únicamente la emoción que comporta el asombro ante el hecho de que él exista; expresa también una disposición para restarse importancia, para retirarse del centro y, por lo tanto, para verse desde el mundo, con él; asume, en fin, una actitud mística.

\section{REFERENCIAS}

Kant, I. (1977). Crítica del juicio. (M. García Morente, trad.). Madrid: Espasa Calpe.

Kant, I. (1983). Werke (Band 8: Kritik der Urteils Kraft und Schriften zur Natur Philosophie). Darmstadt: Wissenschaftlische Buchgesellshaft. Tugendhat, E. (2003). Egozentrizität un Mystik. München: Verlag C.H. Beck.

Tugendhat, E. (2004). Egocentricidad y mística. (M. Suárez, trad.). Barcelona: Gedisa.

Tugendhat, E. (2007). Antropología en vez de metafisica. (D. Gamper, trad.). Barcelona: Gedisa.

Wittgenstein, L. (1999). Conferencia sobre ética con dos comentarios sobre la teoría del valor. (F. Birulés, trad.). Barcelona: Paidós. 